\title{
Genetic engineering of platelets to neutralize circulating tumor cells
}

Jiahe $\mathrm{Li}^{1,2}$, Charles C. Sharkey ${ }^{1}$, Brittany Wun ${ }^{1}$, Jane Liesveld ${ }^{3}$, Michael R. King ${ }^{1 *}$

${ }^{1}$ Department of Biomedical Engineering

Cornell University,

Ithaca, NY, USA, 14853

${ }^{2}$ Current address:

Department of Chemical Engineering

Massachusetts Institute of Technology

Cambridge, Massachusetts, USA, 02139

${ }^{3}$ Department of Medicine, Hematology/Oncology (SMD)

University of Rochester Medical Center

School of Medicine and Dentistry

Rochester, NY, USA, 14642

${ }^{*}$ Correspondence:

Michael R. King, 205 Weill Hall, Ithaca, NY 14853. Phone: 607-255-9803; E-mail:

mike.king@cornell.edu

Key words: circulating tumor cells, platelet, cancer therapy, metastasis

Running title: Killing of CTCs by TRAIL-expressing platelets 


\begin{abstract}
Mounting experimental evidence demonstrates that platelets support cancer metastasis. Within the circulatory system, platelets guard circulating tumor cells (CTCs) from immune elimination and promote their arrest at the endothelium, supporting CTC extravasation into secondary sites. Neutralization of CTCs in blood circulation can potentially attenuate metastases to distant organs.

Therefore, extensive studies have explored the blockade of platelet-CTC interactions as an anti-metastatic strategy. Such an intervention approach, however, may cause bleeding disorders since the platelet-CTC interactions inherently rely on the blood coagulation cascade including platelet activation. On the other hand, platelets have been genetically engineered to correct inherited bleeding disorders in both animal models and human clinical trials. In this study, inspired by the physical association between platelets and CTCs, platelets were genetically modified to express surface-bound tumor necrosis factor-related apoptosis-inducing ligand (TRAIL), a cytokine known to induce apoptosis specifically in tumor cells. The TRAIL-expressing platelets were demonstrated to kill cancer cells in vitro and significantly reduce metastases in a mouse model of prostate cancer metastasis. Our results suggest that using platelets to produce and deliver cancer-specific therapeutics can provide a Trojan-horse strategy of neutralizing CTCs to attenuate metastasis.
\end{abstract}

\title{
Introduction
}


Metastasis contributes to more than $90 \%$ of cancer-associated mortality [1, 2]. It occurs after primary tumors shed circulating tumor cells (CTCs) via hematogenous dissemination to distant organs [3, 4]. Despite advancements in the understanding and detection of CTCs, effective neutralization of CTCs for the prevention of metastasis remains clinically challenging. Previous studies have demonstrated intrinsic tumor-tropic properties of bacteria and multiple types of stem cells including mesenchymal stem cells (MSCs), neural stem cells (NSCs) and endothelial precursor cells, making them attractive candidates for the targeted delivery of anticancer biological agents [5-8]. The mechanisms of tumor tropism were found to be multifactorial including, but not limited to, gradients of hypoxia, growth factors, and inflammatory cytokines generated within solid tumors $[9,10]$. The distinct transport system in the blood circulation, however, allows for rapid exchange of blood components within the vasculature. We reason that the gradients found in solid tumors are absent in circulation, which in turn makes existing cellular engineering approaches ineffective for targeting CTCs.

To explore alternative vectors for the delivery of cancer therapeutics in circulation, platelets were selected since they are capable of recognizing and interacting with CTCs immediately after the release of CTCs into circulation [11, 12]. Platelets are anuclear cytoplasmic bodies released from megakaryocytes in the bone marrow. It is estimated that one liter of blood contains about 400 billion circulating platelets [13]. In contrast to the long history of studies on the 
hemostatic function of platelets, their role in cancer metastasis has only recently become appreciated. It is generally believed that platelets interact with CTCs and promote metastasis via multiple mechanisms: 1. Platelet-CTC aggregates have a greater potential to become trapped in microvessels than individual CTCs. Such aggregation facilitates subsequent extravasation of cancer cells [14]; 2 .

Aggregation of platelets around CTCs protects against immune-mediated clearance of CTCs largely by natural killer (NK) cells [15]; 3. CTC evasion of NK cells is not merely attributed to physical shielding of platelets. The cytotoxic activity of NK cells is guided by the principles of "missing-self" and "induced-self". Cells lacking expression of MHC class I (missing-self) and/or a stress-induced expression of ligands for activating NK receptors (induced-self) are preferentially recognized and eliminated [16]. While CTCs are often associated with a lack of MHC class I ligands, platelets can disrupt "missing self" recognition of NK cells by grafting $\mathrm{MHC}$ I class onto CTCs [17].

In light of the harmful association between platelets and CTCs, a variety of antiplatelet drugs have been tested to block platelet-CTC interactions or inhibit platelet activation in preclinical mouse models [18-20]. Anti-platelet therapies, however, may inevitably impair the normal hemostatic function of platelets in the presence of bleeding $[19,20]$. In contrast to existing platelet intervention therapies, an alternative approach was explored in the current study by expressing the cancer cell-killing cytokine, tumor necrosis factor-related apoptosis-inducing ligand (TRAIL) on the surface of platelets while maintaining 
the platelets' normal hemostatic function and cancer cell adhesion ability. TRAIL was selected to be ectopically expressed in platelets for four reasons: (1) TRAIL is abundantly expressed on the surface of natural killer cells and cytotoxic T cells. It is responsible for the tumoricidal activity of these immune cells [21]. (2) TRAIL exerts a tumor cell-specific apoptotic effect by recognizing death receptors (DRs) highly expressed on the surface of cancer cells [22-24]. Previous studies have successfully modified mesenchymal stem cells (MSCs) and neural stem cells (NSCs) to express TRAIL to kill solid tumors in mice $[7,25,26]$. (3) The adhesion and aggregation of platelets to CTCs may facilitate the DR-mediated TRAIL apoptosis signaling by clustering DRs on cancer cells [27-29]. (4) Despite TRAIL resistances exhibited by certain cancer cells in solid tumors, it was found that these cells become more sensitive to TRAIL when they lose attachment to extracellular matrix [30]. The natural detachment of CTCs from a primary tumor likely increases their sensitivity to TRAIL.

To enable platelet-specific TRAIL expression, a platelet-targeted lentiviral transgene approach was utilized in this work through genetically engineering of hematopoietic stem and progenitor cells (HSPCs) followed by bone marrow transplantation (BMT). Such action has been successfully applied to correct genetic bleeding disorders in mice, dogs and certain human clinical trials while also meeting desired safety requirements [31-35]. The self-renewal ability of HSPCs would allow for continuous presence of TRAIL-expressing platelets upon maturation of megakaryocytes and release of platelets into the circulation. This 
approach can potentially enable long-term patrolling and neutralization of CTCs in circulation for the prevention or reduction of metastases.

\section{Materials and methods}

\section{Construction of lentiviral vectors}

A self-inactivating lentiviral vector pFUWG developed by Dr. David Baltimore's group was utilized in the study [36]. The human ubiquitin-C (Ubc) promoter was replaced by megakaryocyte-specific human integrin all $\beta$ promoter via $\mathrm{BamHI}$ and Xbal (NEB, Cambridge, MA, USA). Green fluorescent protein GFP and TRAIL were cloned separately downstream of $\alpha$ ll $\beta$ promoter via Xbal and EcoRI (NEB). All constructs were verified by DNA sequencing.

\section{Cell lines and mice}

MDA-MB-231, PC3, 293T, Dami and MEG-01 cell lines were obtained from American Type Culture Collection (ATCC, Rockville, MD, USA). MDA-MB-231 and 293 T cells were cultured in DMEM (Invitrogen, Grand Island, NY, USA) with 10\% FBS. PC3 were maintained in RPMI (Invitrogen) with 10\% FBS. Dami and MEG-01 were expanded in IMDM (Invitrogen) with 10\% FBS. Six to eight week old NOD SCID gamma (NSG) and C57BL/6 mice were purchased from Jackson Laboratory (Bar Harbor, ME, USA). Mice were housed in a SPF barrier animal facility at Cornell University.

\section{Chemicals and antibodies}


The following chemicals or kits were used for assaying cell proliferation and apoptosis: MTT (AMRESCO, Solon, OH, USA) and TACS ${ }^{\circ}$ Annexin V-FITC Kit (Gaithersburg, MD, USA). Reagents for TEM were obtained from Electron Microscopy Sciences (Hatfield, PA, USA): glutaraldehyde, osmium tetroxide and uranyl acetate. APC-conjugated antibodies for human and mouse CD41 and PEconjugated antibodies for human TRAIL were purchased from Biolegend (San Diego, CA, USA). Primary antibodies for human TRAIL and $\beta$-actin were obtained from PeproTech (Rocky Hill, NJ, USA) and Santa Cruz Biotech (Santa Cruz, CA, USA). HRP-conjugated anti-mouse and anti-rabbit antibodies were from Santa Cruz Biotech.

\section{Flow cytometry}

Cells were detached with enzyme-free Gibco® Cell Dissociation Buffer (Invitrogen) and suspended at a concentration of $5 \times 10^{5}$ cells in $100 \mu \mathrm{L}$ cold PBS/1\% bovine serum albumin (BSA). Fluorescent primary antibodies or isotype control were incubated with cells for 30 min on ice. Following two washes with 1 $\mathrm{mL}$ of PBS, fluorescence measurements were collected using a Guava easyCyte $^{\mathrm{TM}}$ Flow Cytometry (Millipore, Billerica, MA, USA). Data were analyzed using the Flow Express software (De Novo Software, Los Angeles, CA, USA).

\section{Western blotting}

Western blotting was performed as previously described [37]. Briefly, whole cell lysates were prepared and separated using 10\% SDS-PAGE. Membranes were 
incubated with primary antibodies and secondary antibodies diluted at 1:1000.

Immobilized proteins were detected by using a chemiluminescent HRP substrate (Millipore, Billerica, MA, USA).

\section{Cell proliferation assay}

Cell proliferation was assayed by measuring mitochondrial dehydrogenase activity using MTT as the substrate. After treatment, cells were incubated with MTT at a concentration of $0.5 \mathrm{mg} / \mathrm{mL}$, at $37^{\circ} \mathrm{C}$ for $3 \mathrm{hr}$. The purple MTT product was solubilized with DMSO and measured at $570 \mathrm{~nm}$ using a BioTek plate reader (Winooski, VT, USA).

\section{Isolation, lentiviral transduction and in vitro differentiation of HSPCs}

To isolate mouse HSPCs ( (in $\left.^{-}\right)$, bone marrow mononuclear cells (MNCs) were extracted from femurs and tibias of 6-8 week old mice. Following RBC lysis, unwanted cells were targeted with biotinylated antibodies directed against nonhematopoietic stem cells and non-progenitor cells (CD5, CD11b, CD19, CD45R/B220, Ly6G/C(Gr-1), TER119) followed by removal with streptavidincoated magnetic particles (STEMCELL TECHNOLOGIES INC, Vancouver, BC, Canada). For lentiviral transduction, mouse Lin ${ }^{-}$cells were cultured for 24-hr prestimulation in serum-free X-VIVO-10 (Lonza, Allendale, NJ, USA) containing $100 \mathrm{ng} / \mathrm{mL}$ mouse SCF, TPO, and flt3 ligand (Prospecbio, East Brunswick, NJ, USA). Cells were then transduced twice within $24 \mathrm{hr}$ with lentivirus particles at a 
MOI of 50-100 in retronectin-coated plates. For in vitro differentiation, cells were differentiated in X-VIVO-10, 10\% FBS, and $50 \mathrm{ng} / \mathrm{mL}$ mouse TPO for 10 days.

\section{Bone marrow transplantation (BMT)}

All mice were handled according to the Guide for the Care and Use of Laboratory Animals in compliance with US- and UK-based guidelines. All experimental procedures and protocols were approved by the Institutional Animal Care and Use Committee of Cornell University (Protocol No. 2012-0113). For bone marrow transplantation, 6-8 week old mice were irradiated at a dosage of $2.5 \mathrm{~Gy}$. Within $24 \mathrm{hr}$ of irradiation, each mouse received 1 million transduced HSPCs via retroorbital injection.

\section{Experimental metastasis mouse model with intra cardiac injection}

4 weeks after BMT, NSG mice received $10^{4}$ cancer cells expressing firefly luciferase through intra cardiac injection via left ventricle. Prior to the injection, mice were anesthetized by $2.5 \%$ isoflurane. Anesthetized animals were injected with $1 \times 10^{4}$ cancer cells suspended in $100 \mu$ sterile DPBS containing $300 \mu \mathrm{g} / \mathrm{ml}$ D-luciferin into the left ventricle of the heart by nonsurgical means via a $29 \mathrm{G}$ needle (BD Biosciences). After injection, mice were subject to bioluminescence imaging. A full-body luminescence signal immediately after injection indicated successful left ventricle injection while a localized luminescence signal in the chest suggested injection failure. 


\section{Bioluminescence imaging}

Bioluminescence imaging (BLI) was utilized to longitudinally monitor tumor progression. Briefly, BLI was performed with a CCD camera mounted in a lighttight specimen box (Xenogen, Waltham, MA, USA). Imaging and quantification of signals were controlled by the acquisition and analysis software Living Image $\circledast$ (Xenogen). Anesthetized mice were placed in the IVIS ${ }^{\mathrm{TM}}$ Imaging System and imaged from ventral views approximately $10-15$ min after intraperitoneal injection of D-luciferin at $150 \mathrm{mg} / \mathrm{kg}$ body weight.

\section{Platelet activation}

Murine C57BL6-derived haematopoietic stem and progenitor cells (HSPCs) were transduced with lentiviral transgene and subjected to TPO-induced differentiation for 10 days in vitro. Platelets released into the culture supernatant were collected by centrifugation, activated by $10 \mathrm{nM}$ thrombin for $30 \mathrm{~min}$ and fixed by $4 \%$ paraformaldehyde to prevent spontaneous activation during platelet preparation. Naïve platelets were isolated from whole blood of C57BL6 mice via centrifugation and subjected to the same procedure for thrombin treatment. P-selectin expression on the surface of platelets were examined by flow cytometry with antiP selectin antibody (Biolegend).

\section{Tail bleeding assay}


Tail bleeding time was determined by removing $3 \mathrm{~mm}$ from the tip of the distal mouse tail and immediately immersing the tail in $37^{\circ} \mathrm{C}$ PBS. A complete cessation of bleeding was defined as the end point of bleeding time.

\section{Statistical analysis}

All statistical analyses were performed using GraphPad Prism 5.0a for Mac OS X (San Diego, CA, USA). A one-way ANOVA followed by Tukey post test was used to compare statistical significance in the characterization of in vitro cell proliferation and in vivo experiments.

\section{Results}

Construction of lentiviral vectors with megakaryocyte-specific promoter A self-inactivating lentiviral vector was utilized in this work due to its reported safety in gene therapies for both preclinical studies and clinical trials. To enable megakaryocyte- and platelet-specific gene expression, the human ubiquitin-C (Ubc) promoter in a self-inactivating lentiviral vector (pFUGW) was replaced by human integrin $\alpha$ ll $\beta$ promoter (nucleotides -889 to +35 ) [36, 38]. EGFP was cloned downstream of Ubc or all $\beta$ promoter as a reporter for examing gene transduction efficiency and tissue-specific expression. In addition, a full-length TRAIL gene including transmembrane domain, substituted EGFP for expression of apoptosis-inducing cytokine in megakaryocyte and platelet lineages (Figure 1a). Following lentiviral transduction for $48 \mathrm{~h}$, flow cytometry measurement of 
gene expression indicated that both Ubc and $\alpha / l \beta$ promoters facilitated robust EGFP and TRAIL expression in the promegakaryocyte cell line Dami. In contrast, only Ubc was able to drive gene expression in a human embryonic kidney cell line 293T (Figure 1b and Figure S1). This confirmed the megakaryocyte-specific activity of allß promoter. Immuno-staining of TRAIL in Dami and 293T cell lines transduced with all $\beta$-TRAIL or Ubc-TRAIL indicated surface-bound expression of TRAIL except for allß-TRAIL in 293T (Figure 1c). 
a

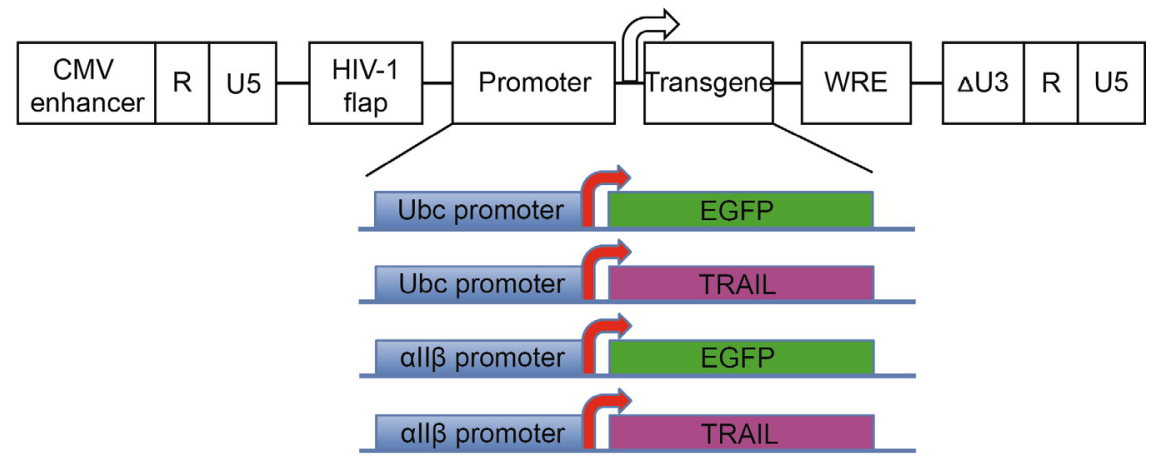

b
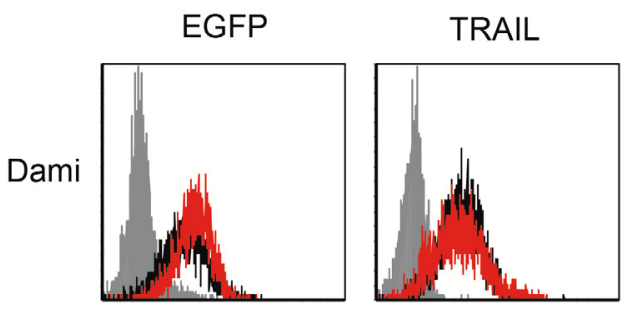
- Nontransduced
- all $\beta$ promoter
- Ubc promoter
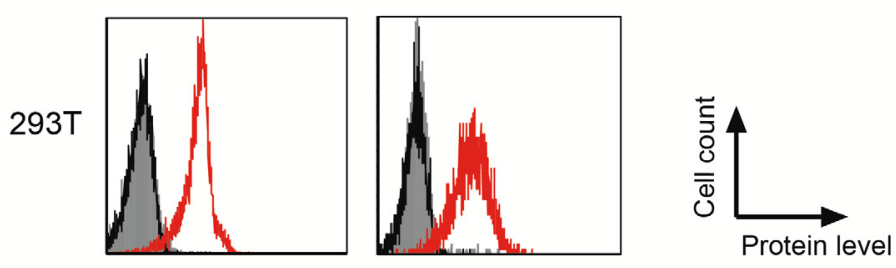

C

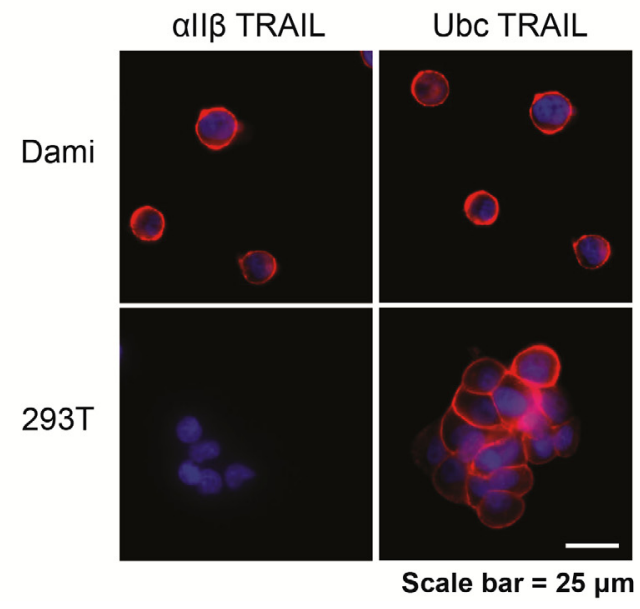

Figure 1. Construction and validation of megakaryocyte-specific lentiviral

expression vectors. (a) Schematic of lentiviral vectors for expression of EGFP and TRAIL in megakaryocytes. (b) Flow cytometry detection of transgene expression after transduction of Ubc-EGFP, $\alpha$ II $\beta$-EGFP, Ubc-TRAIL and $\alpha$ II $\beta$-TRAIL in 
promegakaryocytic cell line Dami and human embryonic kidney cell line 293T, respectively. (c) Immunofluorescence staining of surface-bound TRAIL in Ubc-TRAILand $\alpha$ II $\beta$-TRAIL-transduced Dami and 293T cells. Nuclei were stained with DAPI (blue).

\section{Differentiation of promegakaryocytes upregulates transgene expression in}

\section{a all $\beta$ promoter-dependent manner}

Previous studies showed that the activity of $\alpha$ ll $\beta$ integrin promoter increased following differentiation of promegakaryocytes $[39,40]$. We reasoned that this differentiation pathway could be utilized to produce high levels of membranebound TRAIL. To test this hypothesis, $\alpha$ ll $\beta$-EGFP and $\alpha$ Il $\beta$-TRAIL were transduced into Dami cells. As shown by flow cytometry, expression of EGFP or surface-bound TRAIL was upregulated upon differentiation of Dami by phorbol 12-myristate 13-acetate (PMA) for $48 \mathrm{hr}$. In contrast, Ubc-driven transgene expression was unaffected by PMA-induced differentiation (Figure 2a, $\mathbf{b}$ and Figure S2). In addition, total TRAIL expression also increased following PMA treatment in Dami cells transduced with all $\beta$-TRAIL but not Ubc-TRAIL (Figure 2c). This indicates that robust TRAIL expression can be induced following promegakaryocyte differentiation in an all $\beta$ promoter-dependent manner. 
a

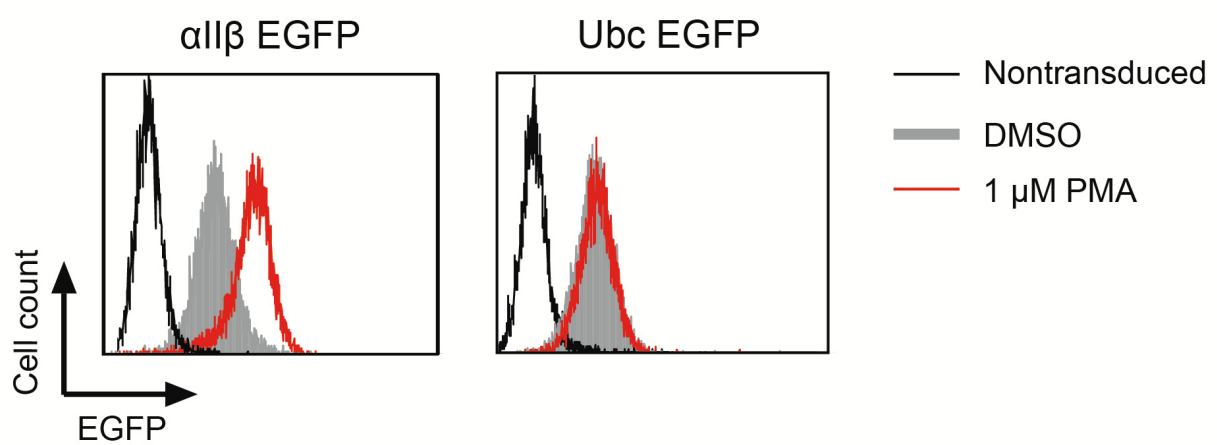

b

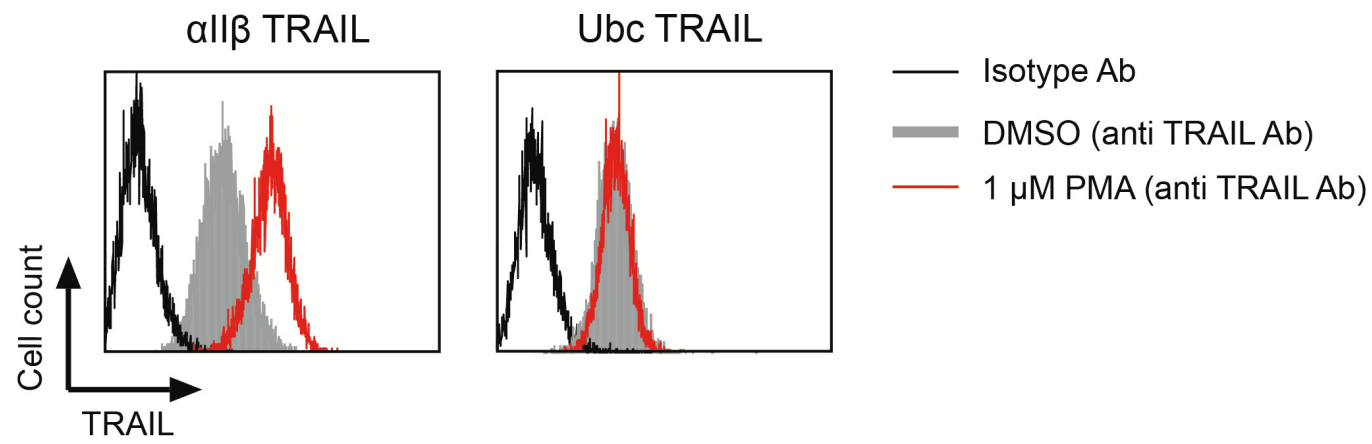

C

\begin{tabular}{|c|c|c|c|}
\hline \multicolumn{2}{|c|}{$\alpha$ II $\beta$ TRAIL } & \multicolumn{2}{|c|}{ Ubc TRAIL } \\
\hline DMSO & PMA & DMSO & PMA \\
\hline
\end{tabular}

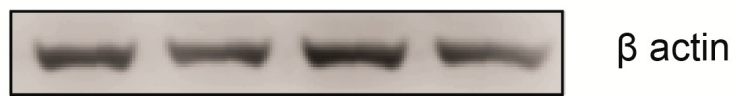

Figure 2. Differentiation of promegakaryocytes increases transgene expression.

Histograms of (a) EGFP and (b) TRAIL expression driven by $\alpha$ II $\beta$ promoter after differentiation of the promegakaryocytic cells, Dami with $1 \mu \mathrm{M}$ PMA for $48 \mathrm{hr}$. In comparison, Ubc-driven transgene expression was not changed upon cell differentiation.

(c) Western blotting of increased total TRAIL expression in $\alpha I I \beta$-TRAIL-transduced Dami cells after PMA-induced differentiation. In contrast, Ubc-TRAIL-transduced Dami showed no change in TRAIL expression after PMA treatment. 


\section{Expression of TRAIL in isolated platelet-like particles}

Dami and Meg-01 cells are two cell lines of megakaryoblastic origin, and are capable of spontaneously producing platelet-like particles (PLPs) [41, 42]. Consistent with previous observation, Dami cells proliferated faster, but produced fewer PLPs than Meg-01 cells (data not shown) [43]. Therefore, PLPs derived from promegakaryocytic cell line MEG-01 were examined in this study. An established protocol via differential centrifugation was adapted to isolate PLPs

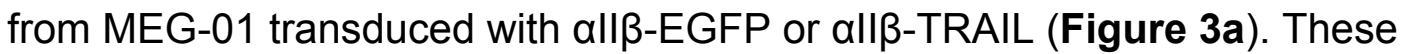
particles were found to be positive for $\beta$-actin detected by western blotting (Figure 3b). This suggested the preservation of cytoskeletal structures in PLPs similar to that of natural platelets. TRAIL-positive particles, however, can only be isolated from cells transduced with all $\beta$-TRAIL but not $\alpha$ II $\beta$-EGFP vectors (Figure 3b). Both flow cytometry and immunostaining demonstrated expression of membrane-bound TRAIL on the surface of PLPs isolated from TRAIL-transduced MEG-01 cells (Figure 3c, $\mathbf{d}$ and Figure S3). Additionally, TEM examination and comparison between $\alpha$ II $\beta$-EGFP- and $\alpha$ II $\beta$-TRAIL-derived PLPs revealed no difference in particle morphology (Figure 3e). 
a

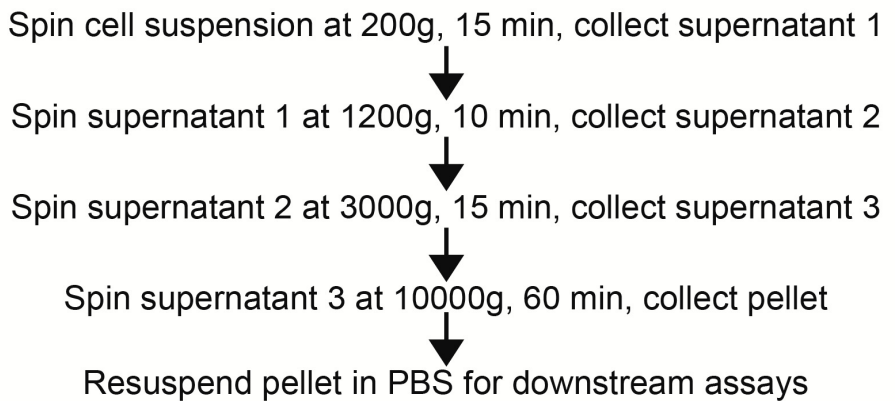

b

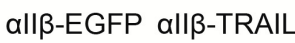

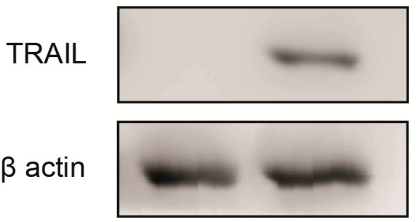

C

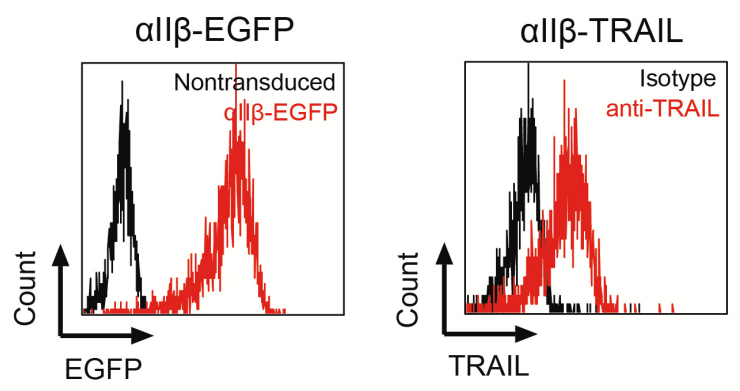

d

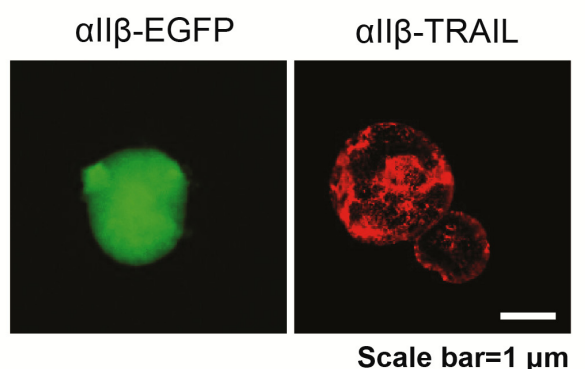

e

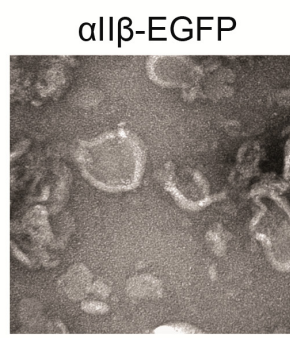

allß-TRAIL

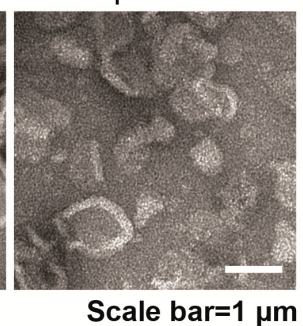

Figure 3. Characterization of PLPs derived from promegakaryocytes. (a) Procedure

for isolating PLPs from MEG-01 cells. (b) Western blotting of total TRAIL and $\beta$-actin in PLPs from PMA-differentiated MEG-01 cells transduced with $\alpha$ II $\beta$-EGFP or $\alpha I I \beta$ -

TRAIL. (c) Flow cytometry and (d) Fluorescence microscopy detection of intracellular EGFP or surface-bound TRAIL in PLPs derived from MEG-01 cells transduced with 
$\alpha I I \beta$-EGFP or $\alpha I I \beta$-TRAIL. (e) TEM imaging of PLPs from culture supernatant of MEG01 transduced with $\alpha I I \beta$-EGFP or $\alpha$ II $\beta$-TRAIL.

\section{Reduction of cancer cell viability by TRAIL-expressing megakaryocytes and PLPs}

Triple-negative breast cancer (TNBC) subtypes and prostate cancer (PCa) bone metastasis represent some of the most deadly forms of metastatic cancer [44, 45]. To explore the possibility of targeting these subtypes, TNBC cell line MDAMB-231 and PCa bone metastatic cell line PC3 were selected as target cells in co-culture experiments. Expression of TRAIL receptors (DR4 and DR5) on these two cancer cell lines was detected by flow cytometry (Figure S4). Following transduction with empty vector control or all $\beta$-TRAIL lentiviral vectors, the promegakaryocytic cells, Dami were incubated with Calcein-AM-labeled cancer cells at a 1:1 or 5:1 ratio (effector-to-target ratio). Dead cells release their Calcein-AM dye into the medium, whereas viable cells retain their fluorescence and can be quantified using flow cytometry. It was found that the viability of both MDA-MB-231 and PC3 cells deceased with increasing effector-to-target cell ratio. Moreover, differentiation with PMA for $48 \mathrm{hr}$ prior to co-culture experiments enhanced cytotoxicity of $\alpha$ II $\beta$-TRAlL-transduced Dami cells against cancer cells (Figure 4a and b). Since TRAIL-R signaling activation induces the deathinducing signaling complex or DISC followed by conversion of pro-caspase 3 (inactive form) to caspase 3 (active form), we used active caspase 3-specific antibody to detect this conversion. After incubating all $\beta$-TRAIL-transduced Dami 
with cancer cells (PC3 and MDA-MB-231), cleavage of pro-caspase 3 was detected by western blotting (Figure S5).

In hematopoietic system, megakaryocytes reside in bone marrow and only platelets shed from megakaryocytes can potentially interact with CTCs in blood circulation. As an in vitro model for platelets, PLPs isolated from TRAILtransduced promegakaryocytic cell lines were utilized to investigate their capability of killing cancer cells in vitro. To this end, PLPs were measured by the total quantity of proteins using the Bradford assay. PLPs with adjusted concentrations were incubated with cancer cells for $24 \mathrm{hr}$. It was found that PLPs from TRAIL-transduced promegakaryocytic cells demonstrated cytotoxicity against cancer cells in a dose-dependent manner (Figure 4c). 

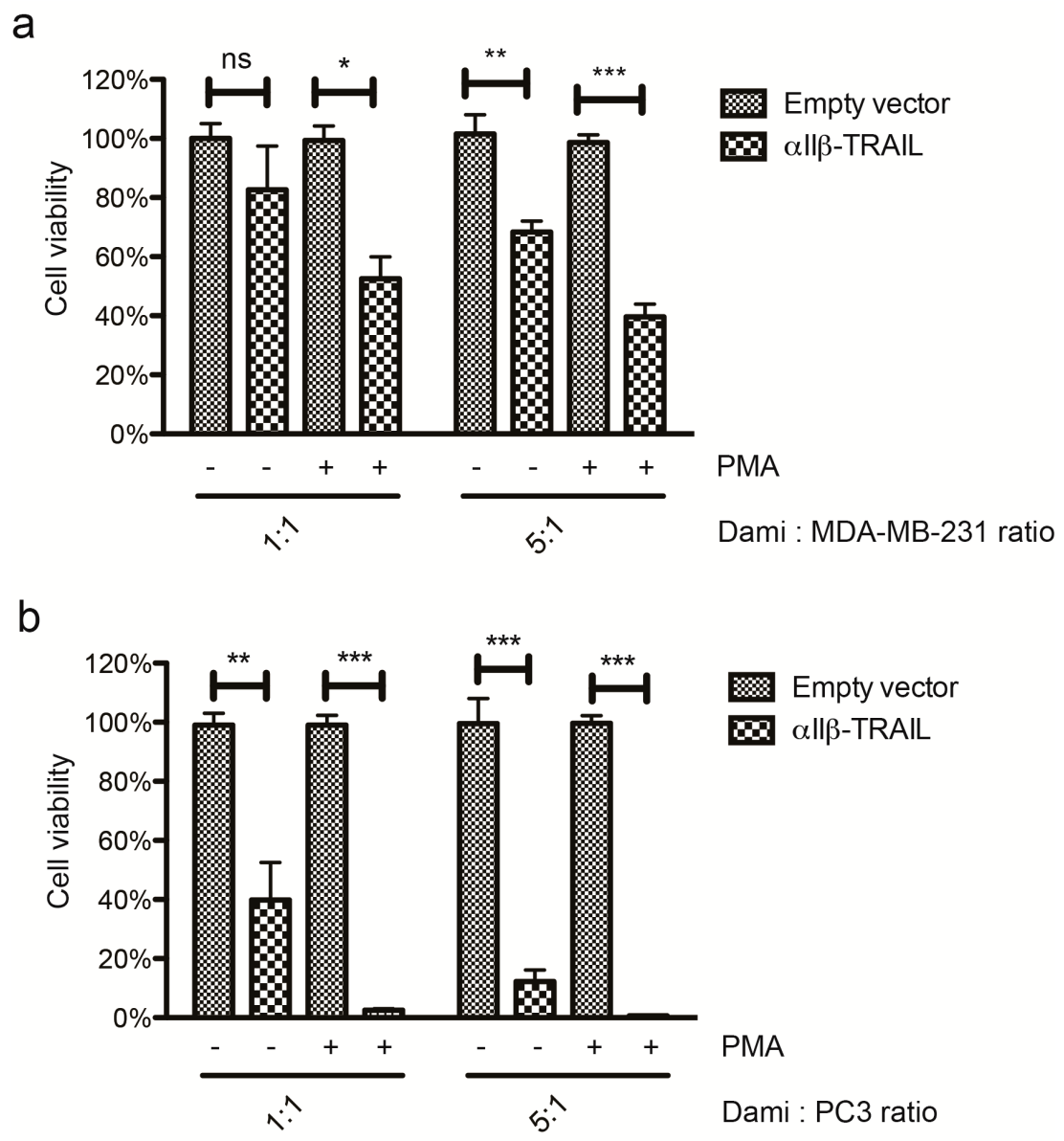

C

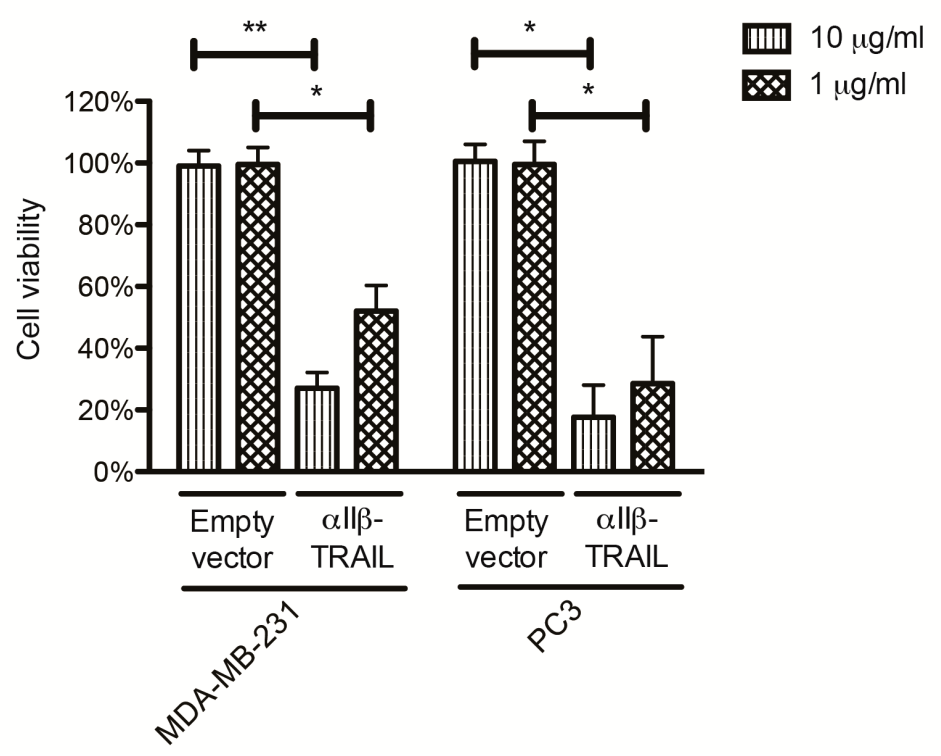

Figure 4. TRAIL-expressing megakaryocytes or PLPs reduce the viability of

epithelial cancer cells in vitro. $\alpha$ II $\beta$-TRAIL-transduced Dami cells were incubated with 
Calcein AM-labeled (a) MDA-MB-231 and (b) PC3 at 1:1 or 5:1 ratio for $24 \mathrm{hr}$ before evaluation of cancer cell viability by flow cytometry. Pretreatment of Dami cells with PMA increased cytotoxicity of $\alpha$ II $\beta$-TRAIL-transduced Dami against cancer cells.

Results are presented as the mean $\pm \mathrm{SEM} ; \mathrm{n}=3$; ns, no significant difference, ${ }^{*}, \mathrm{p}<0.05$, ${ }^{* *}, \mathrm{p}<0.01,{ }^{* *}, \mathrm{p}<0.001$. (c) Reduction of cancer cell viability by PLPs from TRAILtransduced promegakaryocytic cells. Cancer cells were incubated with 10 and $1 \mu \mathrm{g} / \mathrm{mL}$ PLPs derived from empty vector- or $\alpha$ II $\beta$-TRAIL-transduced cells for $24 \mathrm{hr}$. Cell viability was measured by MTT assay. Results are presented as the mean \pm SEM; $n=3 ; *, p<0.05$, **, $\mathrm{p}<0.01$.

\section{Differentiation of TRAIL-transduced HSPCs to megakaryocytes}

Stable gene transduction combined with a lineage-specific promoter in haematopoietic stem and progenitor cells (HSPCs) takes advantage of selfrenewal capability of stem cells to produce transgene product in a targeted blood cell lineage. C57BL/6-derived HSPCs were isolated after lineage depletion from mononuclear cells in bone marrow. After prestimulation with cytokines for $24 \mathrm{hr}$, HSPCs were transduced twice with all $\beta$-TRAIL lentivirus at a MOI of $\sim 100$ within $24 \mathrm{hr}$. Transduced HSPCs were subjected to differentiation in the presence of TPO for 10 days. Double staining for CD41 and TRAIL indicated 57\% TRAIL expression in the megakaryocyte lineage $\left(\mathrm{CD} 41^{+}\right)$(Figure S6a and Figure S7). Maturation of megakaryocytes was distinguished by their large cytoplasm relative to other lineages under examination using bright field microscopy (Figure S6b). Mature megakaryocytes were further verified by Giemsa Wright staining for 
endomitosis (DNA replication without cell division) in which lobulated nuclei were detectable (Figure S6c).

\section{TRAIL overexpression does not induce apoptosis or affect replication of megakaryocytes}

TRAIL signaling has been found to be involved in apoptosis of not only transformed cells but also certain immune cells for tissue homeostasis [46]. It is important to examine whether overexpression of TRAIL in the megakaryocyte lineage would induce apoptosis. Ten days after differentiation of TRAILtransduced HSPCs in the presence of TPO, cells were stained with annexin-V and PI. Necrotic, late apoptotic and early apoptotic cell populations were doublenegative for annexin-V and PI in empty vector- or TRAIL-transduced cells (Figure S8a). As megakaryocytes mature, they undergo DNA replication without cytokinesis. The DNA content was observed to increase from $2 \mathrm{~N}$ to $4 \mathrm{~N}, 8 \mathrm{~N}$, and up to $16 \mathrm{~N}$ (DNA ploidy) in TPO-differentiated megakaryocytes from ex vivo culture. Each ploidy fraction showed comparable ratio between empty vectorand TRAIL-transduced cells (Figure S8b). Therefore, we may conclude that overexpression of TRAIL is tolerable in the megakaryocyte lineage.

\section{TRAIL expression in platelets of bone marrow transplant recipients} HSPCs from NSG mice were transduced with all $\beta$-TRAIL lentivirus at a MOI of $\sim 100$ and subsequently transplanted into NSG mice that received 2.5 Gy of sublethal irradiation (Figure 5a). TRAIL-expressing platelets were identified as a 
CD $41^{+}$TRAIL $^{+}$population in peripheral blood. It was found that $\sim 40 \%$ of circulating platelets were positive for TRAIL at 4, 8, 12 and 20 weeks after BMT (Figure $5 \mathbf{b}$ and $\mathbf{c}$ ). It was estimated that $10^{9}$ platelets expressed $10-30 \mathrm{ng}$ TRAIL by semi-quantitative western blotting assay (Figure S9). Meanwhile, the average copy number of transgene in the genomic DNA of peripheral blood mononuclear cells was quantified by real-time qPCR. It was found that the average copy number of $\alpha$ II $\beta$-TRAIL cassette per cell measured by the presence of lentiviral LTR sequence in the group of recipients was not significantly different from the group receiving empty vector lentivirus over 20 weeks post BMT (Figure 5d). To examine whether platelet activation is affected by TRAIL, HSPCs were transduced with all $\beta$-TRAIL and subjected to TPO-induced differentiation for 10 days in vitro. Platelets released into the culture supernatant were collected by centrifugation and activated by $10 \mathrm{nM}$ thrombin for $30 \mathrm{~min}$. in TRAIL-expressing platelets, P-selectin expression, a marker for platelet activation, was found to be close to that of naïve platelets isolated from the same strain (Figure S10). To examine whether TRAIL expression in megakaryocyte and platelet lineage interfered with blood coagulation, a tail-bleeding assay was performed in mice receiving empty vector- or allß-TRAIL-transduced HSPCs 8 weeks after BMT. $\alpha$ IIß-TRAIL-transduced mice showed no statistically significant difference in bleeding time compared to age- and sex-matched NSG mice without BMT (Figure 5e). Notably, although we were only able to achieve $\sim 40 \%$ TRAILexpressing platelets in vivo, this does not rule out the possibility that TRAIL may affect the coagulation capability within the TRAIL-expressing platelet 
subpopulation. Nevertheless, the bleeding assay suggests that at least the protocol which gives rise to a mixture of TRAIL-positive and -negative platelet populations does not have an adverse effect on blood clotting.

a

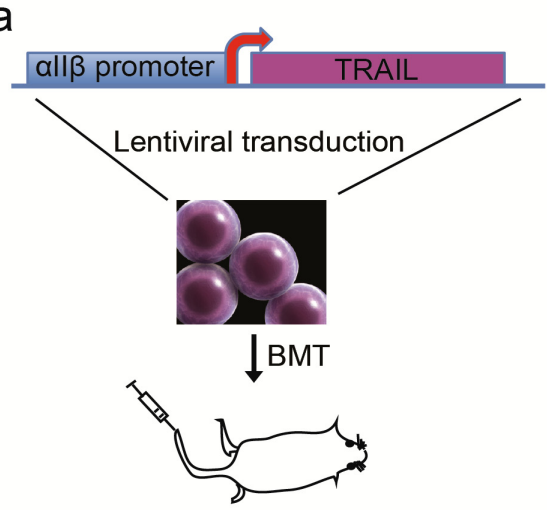

C

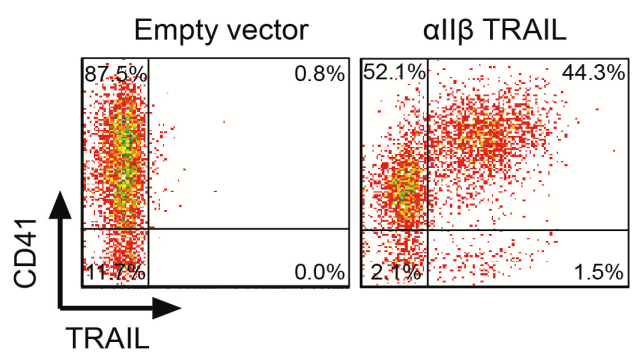

e

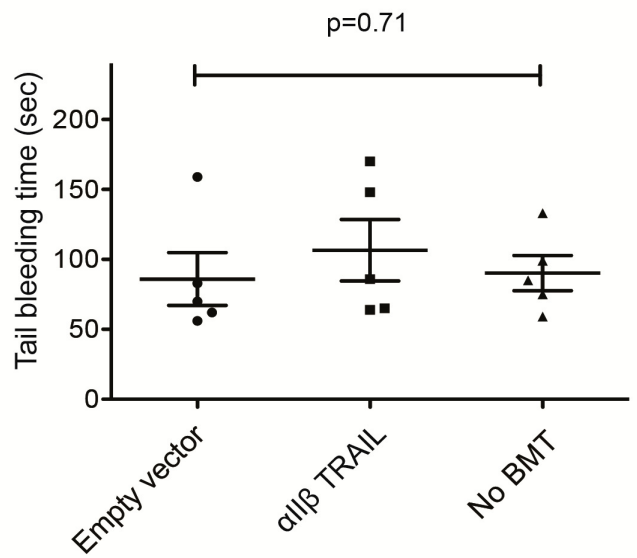

b

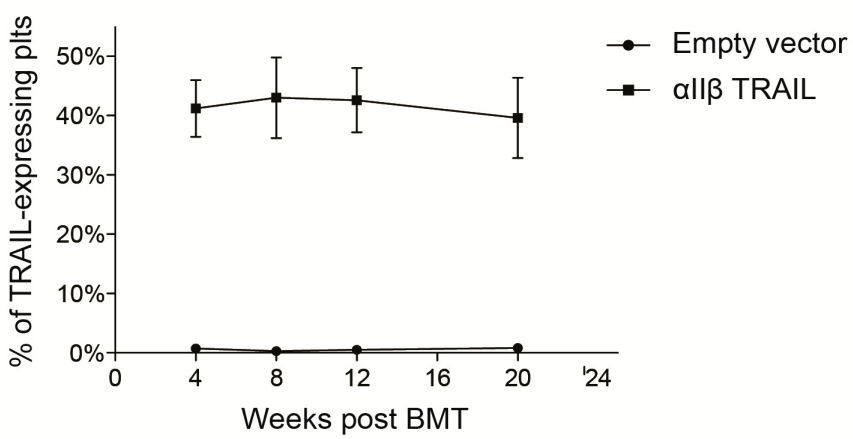

d

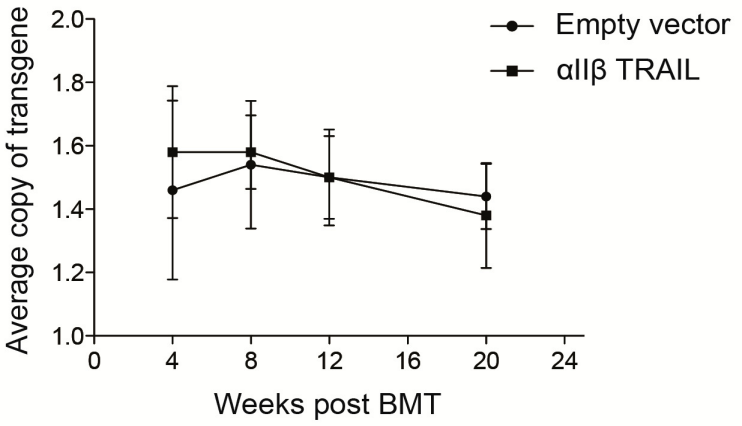

Figure 5. BMT of $\boldsymbol{\alpha I I} \beta$-TRAIL-transduced HSPCs. (a) Schematic of lentiviral transduction of $\alpha$ II $\beta$-TRAIL construct into mouse HSPCs followed by BMT via retroorbital injection. (b) Percentage of TRAIL-positive platelets via flow cytometry in 
peripheral blood of transplanted NSG mice over five months $(n=5)$. (c) Representative dot plots of TRAIL-expressing platelets $\left(\mathrm{CD} 41^{+} \mathrm{TRAIL}^{+}\right)$via flow cytometry 8 weeks after BMT. (d) Quantification of transgene copy number per cell in peripheral mononuclear cells of transplanted NSG mice via qPCR $(n=5)$. (e) Tail-bleeding time of NSG mice 8 weeks after BMT with empty vector- or $\alpha I I \beta$-TRAIL-transduced HSPCs. Age- and sex-matched NSG mice without BMT were used as a control. Results are presented as the mean \pm SEM $(n=5)$. One-way ANOVA was used to test for statistical difference $(\mathrm{p}=0.71)$.

\section{Reduction of cancer metastases via circulating TRAIL-expressing platelets}

To investigate the ability of TRAIL-expressing platelets to reduce distant organ metastases, an experimental bone metastasis model was tested in the study. $10^{4}$ human PCa cells PC3 expressing firefly luciferase were injected into NSG mice via the left ventricle 4 weeks after receiving BMT of $\alpha$ II $\beta$-TRAIL- or empty vectortransduced HSPCs. Progression of PCa metastases was monitored longitudinally by bioluminescence imaging (BLI) (Figure 6a). On day 60 after BMT, BLI revealed that mice with TRAIL-expressing platelets had significantly reduced metastases in the liver relative to control mice receiving empty vector-transduced HSPCs (Figure 6b and c). Upon dissection, metastases were detected mainly in the liver. Relatively large tumor nodules were found in the liver of the control group (Figure 6d). H\&E staining of liver sections showed that the experimental group (TRAIL-expressing platelets) had more localized tumors in liver whereas 
more invasive metastases developed in the control group (Empty vectortransduced platelets) (Figure 6e).

a

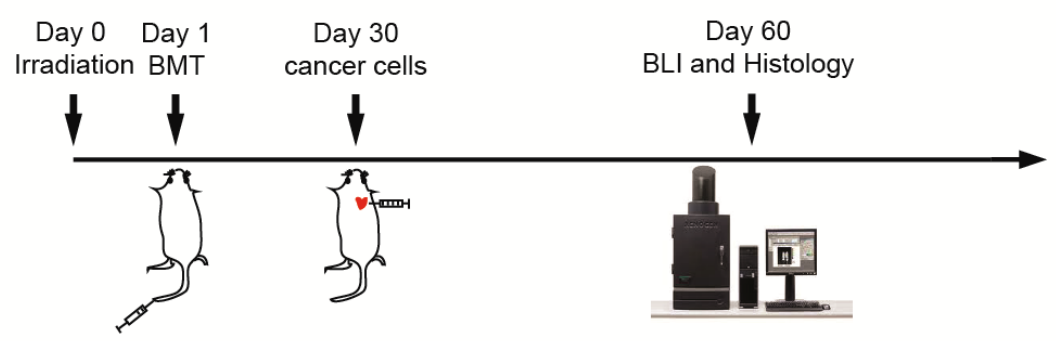

$\mathrm{b}$

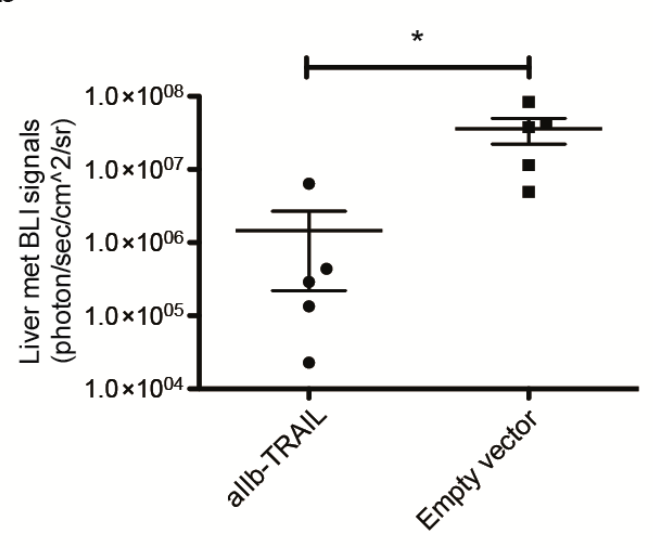

d

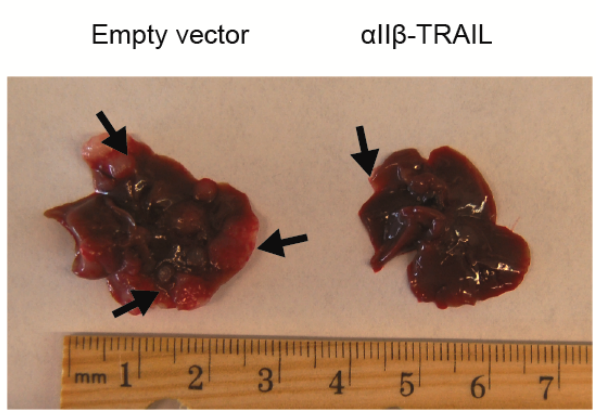

C

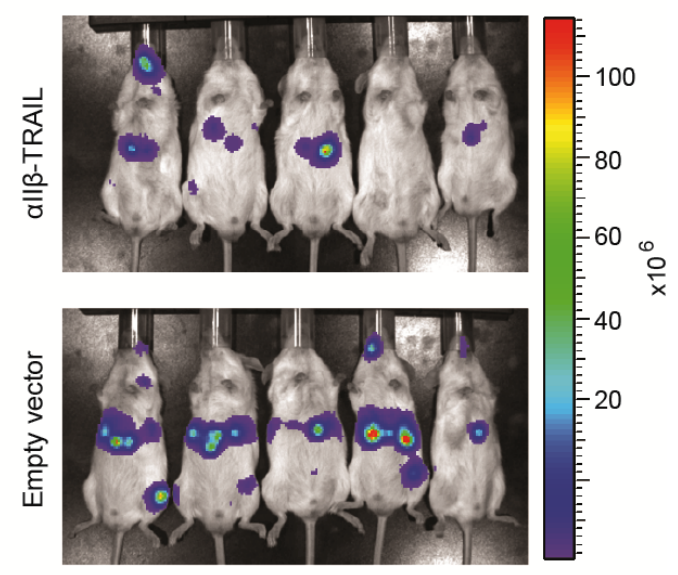

e
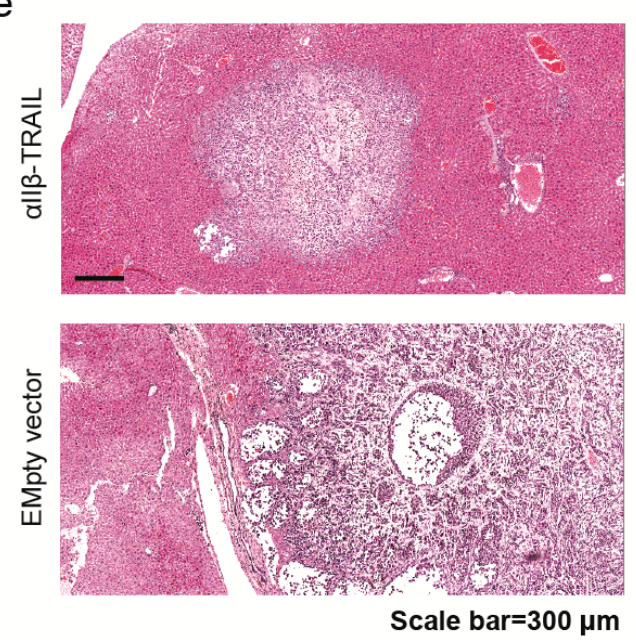

Figure 6. TRAIL-expressing platelets reduced systemic PCa metastases. (a)

Schematic of TRAIL-expressing platelet gene therapy in a PCa experimental metastasis model. (b) Quantification of liver metastatic burden in mice with TRAIL-expressing 
platelets or empty vector-transduced platelets via BLI on day 60 after BMT. Results are presented as the mean \pm SEM, $\mathrm{n}=5,{ }^{*}, \mathrm{p}<0.05$ (c) Representative BLI images of mice on Day 60 after BMT. (d) Liver samples from mice with empty vector-transduced or TRAIL-expressing platelets. Arrows point to white or pink tumor nodules. (e) H\&E staining of liver sections. H: hepatocytes; T: cancer cells.

\section{Discussion}

Thrombocytopenia is often associated with cancer chemotherapy clinically. It causes increased risk of bleeding, reduced dose of chemotherapy, and/or delay of treatment schedules in cancer patients [47]. Management approaches to thrombocytopenia are mainly based on platelet or plasma transfusions [48]. However, the pro-metastatic roles of platelets can possibly counteract the benefits of platelet transfusion $[12,49]$. Nevertheless, this work has demonstrated a proof-of-concept in which platelets were genetically engineered to deliver the cancer cell-specific apoptotic cytokine TRAIL. After the all $\beta$ promoter-driven TRAIL cDNA was inserted into the genome of HSPCs, TRAIL expression was restricted to megakaryocyte and platelet lineages following HSPC differentiation. The self-renewal ability of HSPCs allowed for continual production of TRAIL-expressing platelets in circulation. It was shown that this approach was able to significantly reduce liver metastases in an experimental prostate cancer metastasis model. 
Recently, we published a work that utilized platelet-CTC interactions in the blood to deliver the cancer-killing protein TRAIL to CTCs by coating silica particles with platelet membrane [50]. In addition, other groups have also attempted to functionalize platelet-derived cancer targeting peptides or platelet membrane to the surface of nanoparticles [51,52]. The ability of targeting cancer cells was tested in in vitro cell culture or mouse models bearing solid tumors. These studies collectively suggest utilizing platelet-cancer cell interaction to target both solid and circulating tumor cells. In contrast to these biomimetic approaches, the current study demonstrated an alternative approach of delivering TRAIL via genetic engineering of platelets from haematopoietic stem and progenitor cells (HSPCs). In contrast to previous strategies, the gene therapy method in bone marrow stem cells can potentially avoid repeated administration of cancer drugs such as TRAIL due to the self-renewal ability of HSPCs and stable lentivirusmediated gene transduction. Future work could include engineering a switchable promoter to drive TRAIL expression in response to exogenously administered nontoxic molecules.

The current work showed that the percentage of TRAIL-positive platelets was affected by three factors: (1) dosage of gamma irradiation. The higher the radiation dose (up to $9 \mathrm{~Gy}$ ), the more efficient engraftment of transplanted HSPCs and more TRAIL+ platelets were detected post BMT. Nevertheless, high irradiation has been found to increase post-irradiation mortality rate. Especially in the NSG mouse strain used in this study, a sublethal irradiation dosage (2.5 Gy) 
was applied to minimize post-irradiation mortality. (2) Number of transplanted HSPC. The more HSPCs that were transplanted, the higher the percentage of engrafted HSPCs and reconstituted TRAIL+ platelets found in the circulation post BMT. (3) The ratio of lentiviral vector to HSPC during the viral transduction (i.e. MOI). It was found that a higher MOI (up to 100) led to more transgenic copies of TRAIL in the genome of HSPCs and therefore higher TRAIL expression in the differentiated cells. However, when MOI was too high, genotoxicity of lentiviral vectors could significantly reduce the efficacy of HSPC reconstitution in vivo following BMT.

The current study requires BMT, which can be challenging in certain clinical scenarios [53]. In contrast to the traditional BMT for treating inherited blood diseases, this work requires only autologous HSPCs to be modified. The utilization of autologous HSPCs bypasses the strict criteria for donor-recipient matching. Alternatively, recent studies have indicated that following differentiation of genetically modified HSPCs to megakaryocytes in vitro, functional platelets can be produced upon infusion of megakaryocytes into mice $[54,55]$. This approach can be a future direction for engineering TRAIL-expressing platelets to avoid the complications associated with BMT. In addition to TRAIL, previous studies have identified a variety of tumor-specific killing peptides [56, 57]. A potential direction is to utilize platelets to deliver these different peptides, as appropriate for different cancers. Such an approach can possibly overcome the 
drug resistance from using a single agent for the targeting of CTCs and prevention of metastatic dissemination.

\section{Acknowledgements}

We thank Christine M. Peterson and David E. Mooneyhan for assistance with animal experiments at the Cornell Center for Animal Resources and Education (CARE). This work made use of the Cornell Center for Materials Research Shared Facilities which are supported through the NSF MRSEC program (DMR1120296). We thank Lynn Dong for assistance in imaging IHC slides by ScanScope at the Immunopathology R\&D Lab of Cornell University. This work was supported by $\mathrm{NIH} / \mathrm{NCl}$ grant CA143876 (M.R.K). The authors have no conflicts of interest to disclose.

\section{References}

1. Li, J., et al., Nanobiotechnology for the Therapeutic Targeting of Cancer Cells in Blood. Cellular and Molecular Bioengineering, 2015: p. 1-14.

2. Valastyan, S. and R.A. Weinberg, Tumor metastasis: molecular insights and evolving paradigms. Cell, 2011. 147(2): p. 275-92.

3. Mehlen, P. and A. Puisieux, Metastasis: a question of life or death. Nature reviews. Cancer, 2006. 6(6): p. 449-58.

4. Chambers, A.F., A.C. Groom, and I.C. MacDonald, Dissemination and growth of cancer cells in metastatic sites. Nat Rev Cancer, 2002. 2(8): $p$. 563-72.

5. Massa, P.E., et al., Salmonella engineered to express CD20-targeting antibodies and a drug-converting enzyme can eradicate human lymphomas. Blood, 2013. 122(5): p. 705-14.

6. Stuckey, D.W. and K. Shah, Stem cell-based therapies for cancer treatment: separating hope from hype. Nature reviews. Cancer, 2014. 14(10): p. 683-91. 
7. Kauer, T.M., et al., Encapsulated therapeutic stem cells implanted in the tumor resection cavity induce cell death in gliomas. Nature neuroscience, 2012. 15(2): p. 197-204.

8. Balyasnikova, I.V., et al., Intranasal Delivery of Mesenchymal Stem Cells Significantly Extends Survival of Irradiated Mice with Experimental Brain Tumors. Molecular Therapy, 2014. 22(1): p. 140-148.

9. Nakamizo, A., et al., Human bone marrow-derived mesenchymal stem cells in the treatment of gliomas. Cancer research, 2005. 65(8): p. 33073318.

10. Liu, S.C., et al., Anticancer efficacy of systemically delivered anaerobic bacteria as gene therapy vectors targeting tumor hypoxia/necrosis. Gene Therapy, 2002. 9(4): p. 291-296.

11. Labelle, M. and R.O. Hynes, The Initial Hours of Metastasis: The Importance of Cooperative Host-Tumor Cell Interactions during Hematogenous Dissemination. Cancer discovery, 2012. 2(12): p. 1091-9.

12. Gay, L.J. and B. Felding-Habermann, Contribution of platelets to tumour metastasis. Nature Reviews Cancer, 2011. 11(2): p. 123-134.

13. Daly, M.E., Determinants of platelet count in humans. Haematologica-the Hematology Journal, 2011. 96(1): p. 10-13.

14. Borsig, L., et al., Heparin and cancer revisited: mechanistic connections involving platelets, P-selectin, carcinoma mucins, and tumor metastasis. Proc Natl Acad Sci U S A, 2001. 98(6): p. 3352-7.

15. Nieswandt, B., et al., Lysis of tumor cells by natural killer cells in mice is impeded by platelets. Cancer Res, 1999. 59(6): p. 1295-300.

16. Moretta, L. and A. Moretta, Unravelling natural killer cell function: triggering and inhibitory human NK receptors. EMBO J, 2004. 23(2): p. 255-9.

17. Placke, T., et al., Platelet-derived MHC class I confers a pseudonormal phenotype to cancer cells that subverts the antitumor reactivity of natural killer immune cells. Cancer Res, 2012. 72(2): p. 440-8.

18. Zhang, W., et al., $A$ humanized single-chain antibody against beta 3 integrin inhibits pulmonary metastasis by preferentially fragmenting activated platelets in the tumor microenvironment. Blood, 2012. 120(14): p. 2889-98.

19. Amirkhosravi, A., et al., Assessment of Anti-Metastatic Effects of Anticoagulant and Antiplatelet Agents Using Animal Models of Experimental Lung Metastasis. Anticoagulants, Antiplatelets, and Thrombolytics, Second Edition, 2010. 663: p. 241-259.

20. Hejna, M., M. Raderer, and C.C. Zielinski, Inhibition of metastases by anticoagulants. Journal of the National Cancer Institute, 1999. 91(1): p. 22-36.

21. Wang, S., The promise of cancer therapeutics targeting the TNF-related apoptosis-inducing ligand and TRAIL receptor pathway. Oncogene, 2008. 27(48): p. 6207-15. 
22. Li, J., C.C. Sharkey, and M.R. King, Piperlongumine and immune cytokine TRAIL synergize to promote tumor death. Scientific reports, 2015. 5: p. 9987.

23. Michell, M.J., et al., TRAIL-coated leukocytes that kill cancer cells. Proceedings of the National Academy of Sciences of the United States of America, 2013. 11(3): p. 930-5.

24. Li, J., et al., Targeted drug delivery to circulating tumor cells via platelet membrane-functionalized particles. Biomaterials, 2015. 76: p. 52-65.

25. Leng, Q., et al., TRAIL-secreting mesenchymal stem cells promote apoptosis in heat-shock-treated liver cancer cells and inhibit tumor growth in nude mice. Gene Therapy, 2013. 21(3): p. 317-27.

26. Loebinger, M.R., et al., Mesenchymal Stem Cell Delivery of TRAIL Can Eliminate Metastatic Cancer. Cancer research, 2009. 69(10): p. 41344142.

27. Kopp, H.G., T. Placke, and H.R. Salih, Platelet-derived transforming growth factor-beta down-regulates NKG2D thereby inhibiting natural killer cell antitumor reactivity. Cancer research, 2009. 69(19): p. 7775-83.

28. Wagner, K.W., et al., Death-receptor O-glycosylation controls tumor-cell sensitivity to the proapoptotic ligand Apo2L/TRAIL. Nature medicine, 2007. 13(9): p. 1070-7.

29. Li, J. and M.R. King, Adhesion receptors as therapeutic targets for circulating tumor cells. Frontiers in oncology, 2012. 2: p. 79.

30. Phipps, L.E., S. Hino, and R.J. Muschel, Targeting cell spreading: a method of sensitizing metastatic tumor cells to TRAIL-induced apoptosis. Molecular cancer research : MCR, 2011. 9(3): p. 249-58.

31. Fang, J., et al., Platelet gene therapy improves hemostatic function for integrin alphallbbeta3-deficient dogs. Proceedings of the National Academy of Sciences of the United States of America, 2011. 108(23): $p$. 9583-8.

32. Shi, Q., et al., Lentivirus-mediated platelet-derived factor VIII gene therapy in murine haemophilia A. Journal of Thrombosis and Haemostasis, 2007. 5(2): p. 352-361.

33. Kuether, E.L., et al., Lentivirus-mediated platelet gene therapy of murine hemophilia A with pre-existing anti-factor VIII immunity. Journal of Thrombosis and Haemostasis, 2012. 10(8): p. 1570-1580.

34. Biffi, A., et al., Lentiviral hematopoietic stem cell gene therapy benefits metachromatic leukodystrophy. Science, 2013. 341(6148): p. 1233158.

35. Aiuti, A., et al., Lentiviral hematopoietic stem cell gene therapy in patients with Wiskott-Aldrich syndrome. Science, 2013. 341(6148): p. 1233151.

36. Lois, C., et al., Germline transmission and tissue-specific expression of transgenes delivered by lentiviral vectors. Science, 2002. 295(5556): $p$. 868-72.

37. Li, J., et al., Human fucosyltransferase 6 enables prostate cancer metastasis to bone. British journal of cancer, 2013. 109(12): p. 3014-22.

38. Wilcox, D.A., et al., Integrin alphallb promoter-targeted expression of gene products in megakaryocytes derived from retrovirus-transduced human 
hematopoietic cells. Proceedings of the National Academy of Sciences of the United States of America, 1999. 96(17): p. 9654-9.

39. Fong, A.M. and S.A. Santoro, Transcriptional regulation of alpha Ilb integrin gene expression during megakaryocytic differentiation of K562 cells. Role of a silencer element. The Journal of biological chemistry, 1994. 269(28): p. 18441-7.

40. Zutter, M.M., et al., Regulation of alpha 2 integrin gene expression in cells with megakaryocytic features: a common theme of three necessary elements. Blood, 1995. 86(8): p. 3006-14.

41. Lev, P.R., et al., Production of functional platelet-like particles by the megakaryoblastic DAMI cell line provides a model for platelet biogenesis. Platelets, 2011. 22(1): p. 28-38.

42. Takeuchi, K., et al., Production of platelet-like particles by a human megakaryoblastic leukemia cell line (MEG-01). Experimental cell research, 1991. 193(1): p. 223-6.

43. Sahler, J., et al., A novel method for overexpression of peroxisome proliferator-activated receptor-gamma in megakaryocyte and platelet microparticles achieves transcellular signaling. Journal of thrombosis and haemostasis : JTH, 2012. 10(12): p. 2563-72.

44. Criscitiello, C., et al., Understanding the biology of triple-negative breast cancer. Annals of Oncology, 2012. 23: p. 13-18.

45. Sturge, J., M.P. Caley, and J. Waxman, Bone metastasis in prostate cancer: emerging therapeutic strategies. Nature Reviews Clinical Oncology, 2011. 8(6): p. 357-368.

46. Corazza, N., et al., TRAIL and immunity: more than a license to kill tumor cells. Cell Death and Differentiation, 2004. 11: p. S122-S125.

47. Elting, L.S., et al., Incidence, cost, and outcomes of bleeding and chemotherapy dose modification among solid tumor patients with chemotherapy-induced thrombocytopenia. Journal of Clinical Oncology, 2001. 19(4): p. 1137-1146.

48. Benjamin, R.J. and K.C. Anderson, What is the proper threshold for platelet transfusion in patients with chemotherapy-induced thrombocytopenia? Critical Reviews in Oncology Hematology, 2002. 42(2): p. 163-171.

49. Erpenbeck, L. and M.P. Schon, Deadly allies: the fatal interplay between platelets and metastasizing cancer cells. Blood, 2010. 115(17): p. 34273436.

50. $\mathrm{Li}$, J., et al., Targeted drug delivery to circulating tumor cells via platelet membrane-functionalized particles. Biomaterials, 2016. 76: p. 52-65.

51. Modery-Pawlowski, C.L., et al., A platelet-mimetic paradigm for metastasis-targeted nanomedicine platforms. Biomacromolecules, 2013. 14(3): p. 910-9.

52. Hu, Q., et al., Anticancer Platelet-Mimicking Nanovehicles. Adv Mater, 2015. 27(44): p. 7043-50. 
53. Ringden, O., Allogeneic bone marrow transplantation for hematological malignancies - Controversies and recent advances. Acta Oncologica, 1997. 36(6): p. 549-564.

54. Fuentes, R., et al., Infusion of mature megakaryocytes into mice yields functional platelets. Journal of Clinical Investigation, 2010. 120(11): p. 3917-3922.

55. Lambert, M.P., et al., Challenges and promises for the development of donor-independent platelet transfusions. Blood, 2013. 121(17): p. 33193324.

56. Kawamoto, M., et al., A novel transferrin receptor-targeted hybrid peptide disintegrates cancer cell membrane to induce rapid killing of cancer cells. Bmc Cancer, 2011. 11.

57. Wu, D.D., et al., Peptide-based cancer therapy: Opportunity and challenge. Cancer Letters, 2014. 351(1): p. 13-22. 


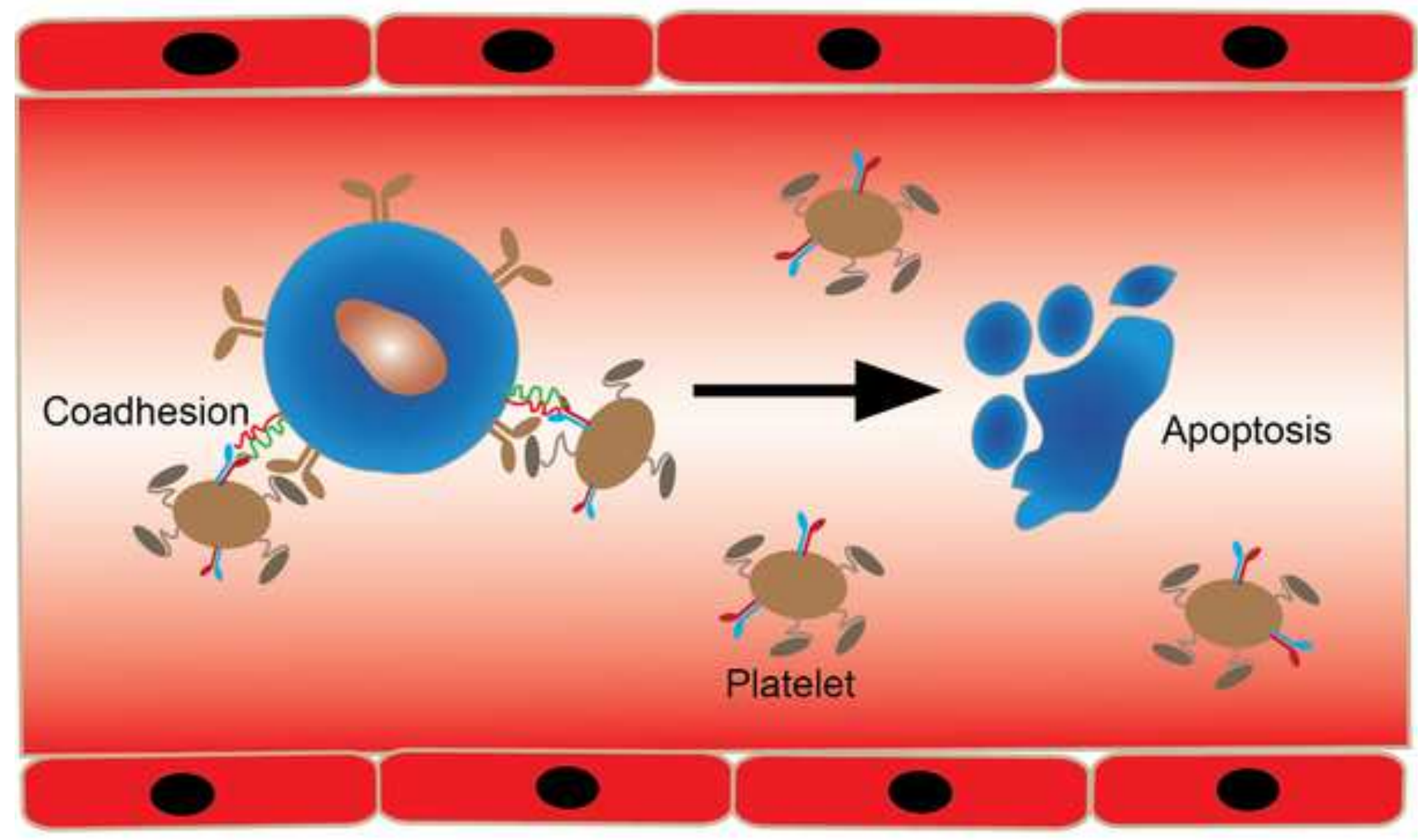

\& TRAIL receptor 9 \& Adhesion receptors

Y TRAIL 